\title{
Une protéine à facettes multiples : le facteur nécrosant des tumeurs (tumor necrosis factor, TNF)
}

Au cours des dernières années, on a mis en évidence un nombre croissant de protéines intervenant dans l'inflammation et l'immunité comme dans la croissance et l'inhibition cellulaires. Ces protéines, auxquelles on a donné le nom général de cytokines, comprennent par exemple les interleukines et les interférons. Parmi elles, c'est le TNF (tumor necrosis factor) qui possède le spectre d'activité le plus étendu. Il ne se passe guère de mois sans qu'on lui décrive une propriété nouvelle et sans qu'une revue générale en présente les diverses facettes [1, 2] ou insiste sur l'une d'entre elles [3]. C'est dire la difficulté de tracer un tableau à la fois clair et suffisamment complet de son action.

Le nom sous lequel est connu le TNF ne reflète que la première propriété qu'on lui a attribuée et qui a mené à sa découverte. Des travaux, dont l'origine remonte au siècle dernier, avaient montré que la nécrose hémorragique de certaines tumeurs de la souris était due à l'action d'une endotoxine bactérienne identifiée comme un lipopolysaccharide (LPS). En même temps, on fonda de grands espoirs sur le BCG comme arme anticancéreuse. En 1975, Carswell et al. [in l] démontrèrent que le sérum de souris ayant reçu BCG et LPS provoque la nécrose hémorragique de sarcomes de souris. Le TNF était né ; restait à le caractériser. Une protéine dissociable en deux sousunités de $17 \mathrm{kDa}$ (kilodalton) fut isolée dans plusieurs espèces. En 1984, Pennica et al[4] clonèrent l'ADN complémentaire du TNF humain; on connaît actuellement la séquence du TNF de plusieurs espèces et on le fabrique par génie génétique. Le TNF recombinant obtenu dans le colibacille est directement actif, car il ne semble pas y avoir de glycosylation. La chaîne de ce TNF appelé
TNF $\alpha$, tel qu'il est sécrété dans le plasma, compte 156 acides aminés chez la souris, 154 chez le lapin et 157 chez l'homme. L'homologie entre les espèces est d'environ $80 \%$. La forme mature dérive d'un précurseur, dont le propeptide a une longueur, inhabituelle pour un peptide signal, de 76 acides aminés chez l'homme et 79 chez la souris. La taille de cette séquence, jointe à une remarquable conservation $(86 \%$ d'homologie entre homme et souris) a fait penser que le propeptide pourrait jouer un rôle biologique propre. Nous y reviendrons plus loin.

Le TNF recombinant reproduisait les trois propriétés connues à cette date: nécrose hémorragique de certaines tumeurs, cytotoxicité vis-à-vis de cellules humaines et murines, synergie avec l'interféron $\gamma$. Mais, dès l'année suivante, l'équipe de Cerami [5] isola à partir de tissus infectés une substance qu'elle nomma cachectine, dont le clonage démontra l'identité avec le TNF. C'était le premier exemple de la découverte, à partir de propriétés entièrement différentes, de deux protéines qui se révèlent n'en faire qu'une $\left(\mathrm{m} / \mathrm{s} n^{\circ} 1\right.$, vol. 2, p. 49).

Localisation génique. Le gène du TNF possède quatre exons et trois introns; il en existe un seul par génome haploïde. Chez l'homme, il se situe sur le bras court du chromosome 6, étroitement lié à celui d'une protéine qui partage la plupart de ses propriétés et qu'on appelle TNF $\beta$ ou lymphotoxine. L'ensemble des deux TNF est lui-même lié au complexe majeur d'histocompatibilité.

Propriétés biologiques. Schématiquement, on peut décrire trois champs d'action principaux au TNF, dont le plus connu, l'effet sur les tumeurs, n'est peut-être pas le plus important. A ces champs principaux s'ajoutent de nombreuses propriétés difficiles à classer.

- Action sur les tumeurs. L'observation originale porte sur la lyse de sarcomes murins et la régression in vivo de ce type de tumeur chez la souris; le sérum de souris contenant du TNF est également toxique in vitro pour les cellules $\mathrm{L}$. La production de facteurs nécrotiques et cytotoxiques exigerait deux étapes: un premier événement, celui qui est déclenché, par exemple, par le BCG, provoque l'activation et la prolifération des macrophages; un deuxième événement («élicitation ») déclenche l'apparition du facteur dans le plasma. L'effet antitumoral, cependant, est loin d'être général. Le TNF n'a pas d'action sur les tumeurs mammaires primitives de la souris, ni sur la plupart des lignées normales; il peut même stimuler la croissance des fibroblastes. Récemment, Cordingley et al. [6] ont même montré que le TNF pouvait provoquer la prolifération de cellules malignes dans deux types de leucémie humaine à cellules $\mathrm{B}$, la leucémie à tricholymphocytes et la leucémie lymphocytaire B chronique. C'est dire à quel point il faudra se montrer prudent lorsqu'on abordera les problèmes thérapeutiques.

- Action sur l'état général: la cachectine. En réponse à certaines infections, le lapin peut perdre jusqu'à $50 \%$ de son poids. Cet amaigrissement s'accompagne d'une hyperlipidémie à triglycérides en rapport avec une inhibition de la lipoprotéine lipase. Beutler et Cerami [5] observèrent ensuite que la suppression de cette enzyme s'observe dans les souches de souris sensibles au LPS, mais non dans les souches résistantes. Le facteur responsable de la cachexie, qui est capable d'inhiber la lipoprotéine lipase dans des adipocytes isolés, fut appelé cachectine. Beutler et Cerami lui attribuent un 
rôle considérable dans les effets de l'endotoxinémie - choc, fièvre. hypotension, acidose métabolique qui peuvent conduire à la mort, et au cours desquels la teneur en TNF du sang s'élève. Il peut être, en fait, très difficile d'évaluer le rôle du TNF in vivo dans la survenue de la cachexie, car les résultats expérimentaux dépendent de la dose et du mode d'administration. Ollif [3] a utilisé une méthode ingénieuse pour provoquer la production, chez la souris nude, de tumeurs sécrétant du TNF. Ces animaux ont effectivement été atteints d'un syndrome analogue à la cachexie associée au cancer.

- Action immunologique. Le TNF agit sur le système immunitaire, soit directement, soit par l'intermédiaire d'autres cytokines (figure 1). Des animaux déficients en production de TNF seraient prédisposés à des maladies auto-immunes. Rappelons également qu'il est capable d'induire les antigènes d'histocompatibilité de classe I dont nous avons vu qu'ils étaient codés par des gènes situés à proximité du gène TNF.

- Autres actions. Elles sont multiples $[7,8]$ et ne peuvent qu'être énumérées sans que l'on puisse prétendre être exhaustif $\left(m / s n^{\circ} 2\right.$, vol. 3 , p. 112): (a) action pyrogène, à la fois par effet direct sur les centres hypothalamiques et par induction de la biosynthèse d'interleukine I ; (b) action sur le tissu articulaire, qui constitue pour le TNF une cible privilégiée: production accrue de prostaglandine E2 par les cellules synoviales, activation de la phospholipase A2 dans les chondrocytes; (c) inhibition de la synthèse du colla. gène et des protéoglycanes dans le tissu osseux, alors que les ostéoclastes sont activés, pouvant être la cause d'une résorption osseuse accélérée ; (d) inhibition de la croissance des cellules endothéliales in vitro, alors qu'in vivo prédomine une action angiogénique $\left(\mathrm{m} / \mathrm{s} n^{\circ} 5\right.$, vol. 4, p. 318); (e) stimulation de l'activité procoagulante par les cellules vasculaires endothéliales.

Mécanismes biochimiques. Ils sont loin d'être tous connus, mais on peut dégager des effets sur certaines enzymes ou voies métaboliques: pase A 2: les inhibiteurs de cette enzyme interfèrent avec de nombreuses actions du TNF, y compris ses propriétés cytotoxiques et antitumorales; (2) inhibition de la lipoprotéine lipase, dont le dosage sert au calibrage biologique de la cachectine; (3) activation du métabolisme de l'acide arachidonique, dont certains métabolites sont toxiques pour divers types de cellules transformées. Ces propriétés ont incité Hepburn $e t$ al. [8] à suggérer qu'une protéine liant le GTP serait impliquée dans l'action toxique du TNF.

Mécanismes cellulaires et moléculaires. Contrairement à d'autres facteurs intervenant dans la croissance normale ou tumorale, le TNF n'est pas ubiquitaire. Il est sécrété avant tout par les lymphocytes et surtout les macrophages, et transporté par voie circulatoire à ses cibles, aux- quelles il se lie sur un récepteur spécifique. Les cellules contiennent de 1000 à 10000 copies du récepteur, qui semble formé de deux chaînes de 75 et $95 \mathrm{kDa}$, mais dont l'analyse n'est pas terminée.

Dans le macrophage avant activation, le messager est présent mais non traduit [5]. L'exposition au LPS déclenche à la fois une augmentation de la transcription, la mobilisation du messager et sa traduction. Les souches de souris résistantes au LPS ont une transcription et une mobilisation diminuées, et ne produisent pas de TNF. Les glucocorticoïdes inhibent toutes les phases de la biosynthèse du TNF, transcription, mobilisation et traduction.

Enfin le TNF est capable de supprimer l'expression d'ARN messagers dans les tissus cibles et pourrait donc

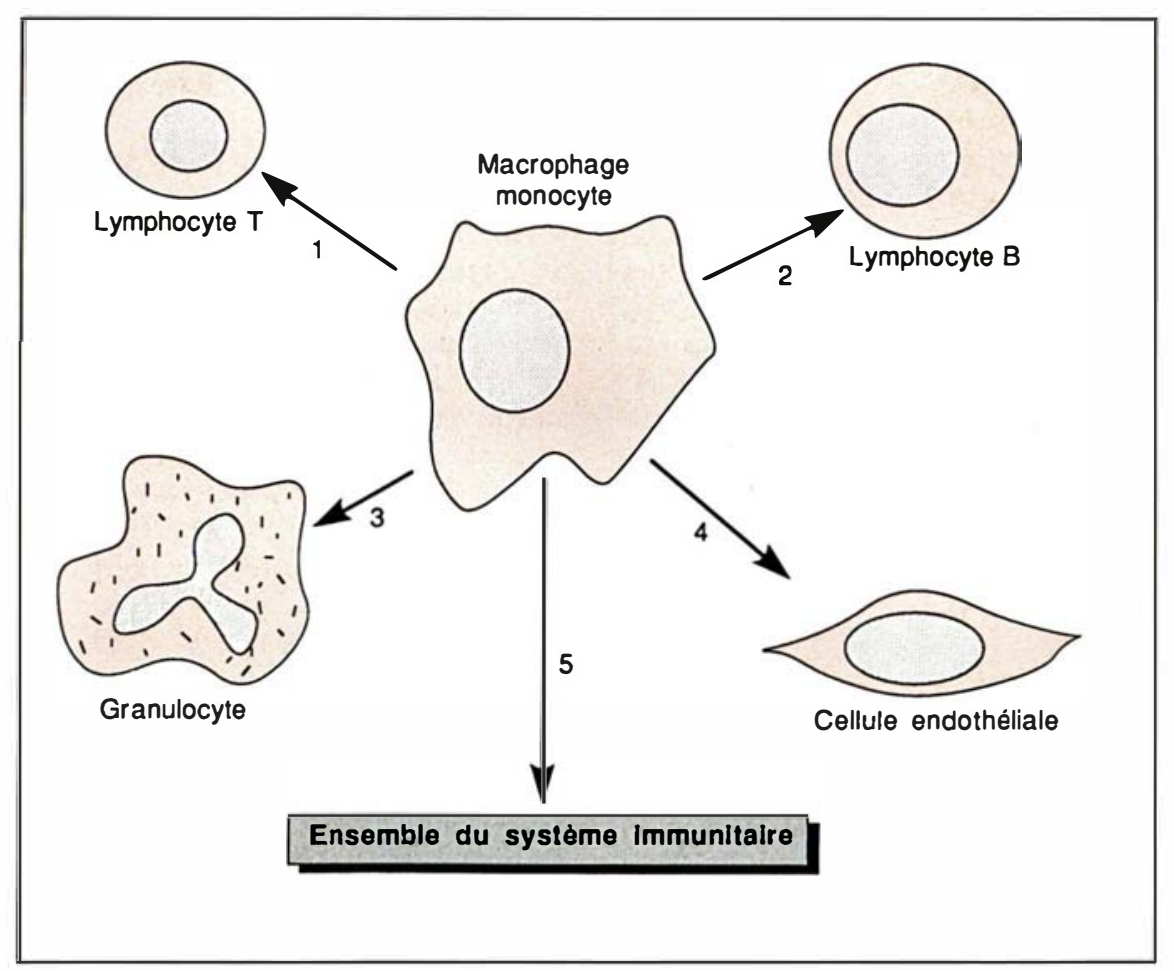

Figure 1. Relations du TNF avec lo système immunitaire. 1. Lymphocytes T: Stimulation de certaines sous-populations. 2. Lymphocytes B : Synthèse d'ADN et d'immunoglobulines. 3. Granulocytes: stimulation de l'adhésion cellulaire et de la migration vers les tissus lésés. 4. Cellules endothéliales: induction de molécules d'adhésion qui peuvent promouvoir le passage hors des tissus. 5. Effet indirect sur l'ensemble du système immunitaire par libération d'autres cytokines (IL-1, IL-6 ou $\gamma$ (NF). 
jouer un rôle dans la différenciation cellulaire.

Analogies et interactions avec d'autres facteurs. Nombreux sont les facteurs avec lesquels peut se coordonner le TNF: Trois d'entre eux font preuve d'une apparente spécificité.

- TNFB ou lymphotoxine. Découvert, comme l'indique son nom, par ses propriétés toxiques sur des cellules lymphoïdes, le TNF $\beta$ est génétiquement lié au TNF $\alpha$ sur le chromosome 6 . Bien que ne présentant entre eux qu'une homologie de $30 \%$, les deux TNF ont des structures très voisines ( 4 exons et 3 introns) et paraissent dériver de la duplication d'un même gène ancestral; leurs séquences régulatrices en 5 ' sont similaires ; leurs activités sont semblables et leurs récepteurs sont communs.

- Interleukine I. Nous avons déjà signalé les synergies entre TNF et IL-I, par exemple l'activation de la phospholipase A2 dans les chondrocytes.

- Les interférons. En particulier INF $\gamma$ dont l'analogie de propriétés avec TNF avait frappé les chercheurs depuis le début.

Rôle du précurseur et conception actuelle du mécanisme d'action. La biosynthèse du TNF mature suit apparemment la voie habituelle d'un produit de sécrétion après élimination de son peptide signal. Cependant cette élimination n'avait pas pu être observée en système acellulaire [9], aussi Kriegler $e$ t al. [10] ontils entrepris la recherche du précurseur sur des monocytes humains. Une heure après exposition de ces cellules au LPS, des anticorps anti-TNF détectent une protéine de $26 \mathrm{kDa}$, qui ne peut être que le précurseur, et qui, en quelques heures, se transforme en TNF 17 kDa. Or il est inhabituel que l'on puisse mettre en évidence le précurseur d'une protéine sécrétée, puisque son peptide signal est coupé au cours même de la traduction. L'analyse de la séquence de 76 acides aminés du propeptide la montre formée d'une portion hydrophobe de 20 acides aminés encadrée de deux séquences hydrophiles, l'ensemble ayant le caractère d'un domaine transmembranaire. Le précurseur $\mathrm{m} / \mathrm{s} n^{\circ} 10$ vol. 4, décembre 88
26 kDa du TNF apparaît ainsi comme une protéine transmembranaire: la partie aminoterminale du propeptide est cytoplasmique, la partie carboxyterminale du TNF, qui porte la cytotoxicité, se trouverait à l'extérieur de la cellule. A la suite de leur travail, Kriegler et al. proposent le modèle suivant (figure 2) : un premier temps comporte l'activation des monocytes qui migrent vers leur cible, par exemple une tumeur ou le siège d'une inflammation. Le deuxième temps voit la synthèse du TNF $26 \mathrm{kDa}$, qui pourrait agir directement ou après transformation en forme $17 \mathrm{kDa}$ sécrétée. Le plus souvent l'action reste locale, notamment dans le cas d'intervention de la forme $26 \mathrm{kDa}$ qui est liée à la membrane. $\mathrm{Si}$, cependant, se développe une activation généralisée des macrophages, l'action cesse d'être locale, choc et cachexie peuvent apparaître, provoqués par la libération de la forme mature de $17 \mathrm{kDa}$.

En terminant ce chapitre, il ne faut pas perdre de vue une hypothèse: peut-être ignorons-nous encore quelle est l'action principale du TNF et de son précurseur, et les travaux les plus importants sont-ils

encore à venir

Perspectives thérapeutiques. Ce sont naturellement les propriétés antitumorales du TNF qui ont soulevé le plus d'espoirs. Les quelques tentatives faites chez des cancéreux ont été bien supportées mais n'ont pas donné de résultats concluants. Il est très difficile de définir une dose active, et, étant donné la très courte demivie du TNF injecté, d'obtenir une action soutenue. Peut-être les tentatives de synergie TNF-INF $\gamma$ aurontelles plus de succès. Si l'on veut, à l'avenir, espérer un traitement antitumoral, il faudra dissocier effets antitumoraux et toxiques par des modifications appropriées de la molécule.

De fait, tout récemment, Frey $e t$ al. [11] ont construit un gène hybride entre interféron $\gamma$ et TNF $\beta$. Ce gène, exprimé dans le colibacille, possède à la fois les actions antivirales et antitumorales. Cette dernière est nettement plus forte que lorsque les deux facteurs intacts sont utilisés conjointement. Les auteurs espèrent trouver à cette découverte des applications thérapeutiques.

Mais c'est peut-être, paradoxalement, par un raisonnement inverse que la

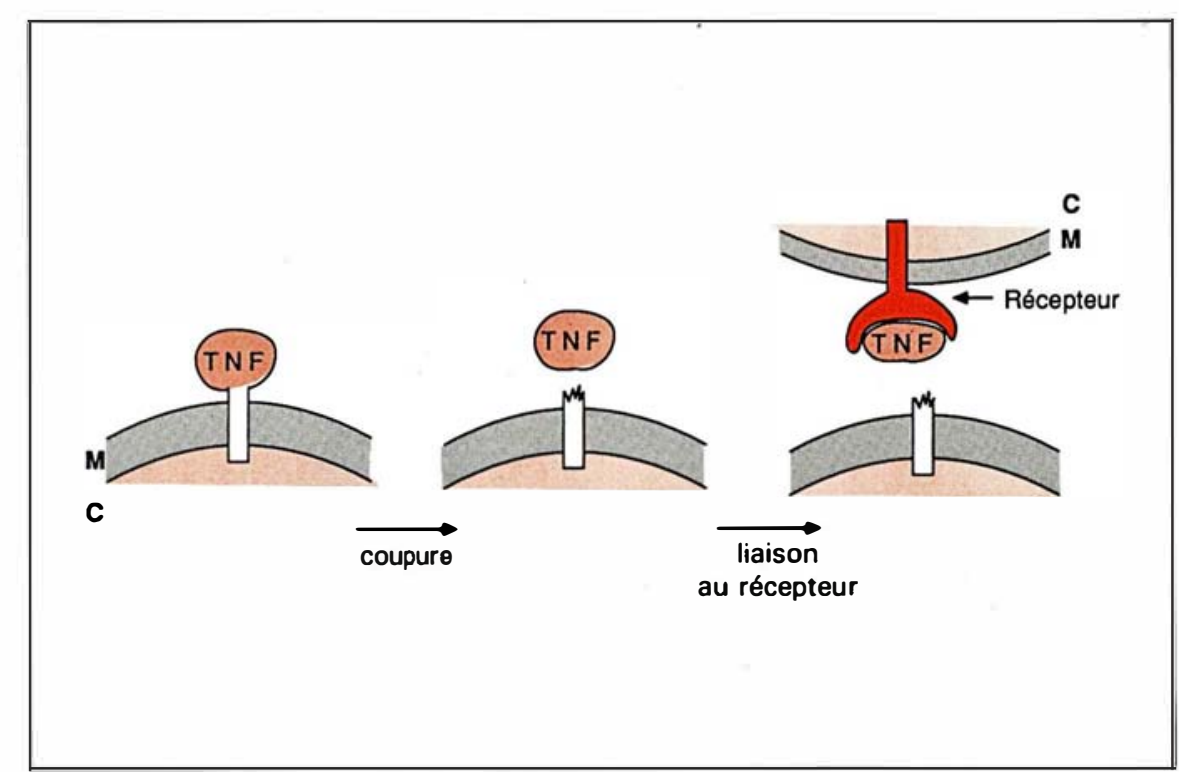

Figure 2. Le précurseur du TNF (tumor necrosis factor) et sa maturation (modifié d'après [10]). $M=$ membrane ; $C=$ cytoplasme. 
connaissance du TNF pourrait mener à des thérapeutiques intéressantes: bien souvent l'action du TNF est nocive, et c'est à l'atténuer qu'il faudrait viser. Cet objectif passe par la découverte d'antagonistes susceptibles d'agir au niveau du récepteur et au-delà, qui pourraient prendre le relais des glucocorticoïdes, seuls inhibiteurs connus, sans en avoir les inconvénients. Ces antagonistes s'adresseraient, non à l'aspect antitumoral, mais à l'effet «cachectine », en luttant contre le choc, l'inflammation et la perte de poids [12].

Jean-Claude Dreyfus

\section{RÉFÉRENCES}

1. Old LJ. Le facteur nécrosant des tumeurs. Pour la Science 1988 ; 129 : 58-66.

2. Brenner MK. Annotation; tumor necrosis factor. Br J Haematol 1988 ; 69 : 159-62.

3. Oliff A. The role of tumor necrosis factor (cachectin) in cachexia. Cell 1988; $54: 141$ 2.

4. Pennica D, Nedwin GE, Hayflick JS, et al. Human tumor necrosis factor, precursor structure, expression and homology to lymphotoxin. Nature 1984 ; 312 : 724-8.

5. Beutler B, Cerami A. Cachectin and tumor necrosis factor as two sides of the same biological coin. Nature 1986 ; 320 : 584-8.

6. Cordingley FT, Bianchi A, Hoffbrand AV, et al. Tumor necrosis factor as an autocrine tumor growth factor for chronic B-cells malignancies. Lancet 1988 ; i : 969-71.

7. Frater-Schröder M, Risal W, Hallmann R, Gautsch P, Böhlen P. Tumor necrosis factor type $\alpha$, a potent inhibitor of endothelial cell growth in vitro, is angiogenic in vivo. Proc Natl Acad Sci USA 1987 ; 84 : 5277-81.

8. Hepburn A, Boeynaems JM, Fiers W Dumont JE. Modulation of tumor necrosis factor cytotoxicity in L 129 cells by bacterial toxins, hydrocortisone and inhibitors of arachidonic acid metabolism. Biochem Biophys Res Commun 1987 : 149: 815-22.

9. Muller R, Marmenout A, Fiers W. Synthesis and maturation of recombinant human tumor necrosis factor in eucaryotic systems. FEBS Lett 1986 ; 197 : 99-104.

10. Kriegler M, Perez C, De Fay K, Albert I, LuSD. A novel form of TNF/cachectin is a cell surface cytotoxic transmembrane protein : ramifications for the complex physiology of TNF: Cell 1988; 53 : 45-53.

11. Feng GS, Gray PV, Shepard HM, Taylor MW. Antiproliferative activity of a hybrid protein between Interferon and tumor necrosis factor. Science 1988; 241 : 1501-3.

12. Sherry B, Cerami A. Cachectin/TNF exerts endocrine, paracrine and autocrine control of inflammatory responses. J Cell Biol

\section{BRËVES}

Thérapie génétique antivirale. L'expression du génome viral, et donc le cycle viral, est sous le contrôle de facteurs de transcription agissant en trans, certains codés par le virus, d'autre par la cellule hôte. Ainsi, les gènes précoces du virus herpes (HSV-l) sont-ils transcriptionnellement activés par la protéine virale VP16 dont l'extrémité carboxyterminale, à caractère acide, est indispensable à la fonction. Des cellules transfectées par une construction commandant la synthèse d'une protéine VPl6 dont l'extrémité carboxyterminale a été délétée deviennent résistantes au virus HSV-l dont les gènes précoces ne sont plus exprimés. Il est probable que la protéine modifiée entre en compétition avec VP16 sauvage pour la liaison aux séquences activatrices d'ADN viral [1]. Envisagera-t-on demain de recombiner in vitro des cellules médullaires humaines avec un gène tat modifié afin que, réimplantées, elles deviennent résistantes au virus du SIDA ? [2]

[1. Friedman AD, et al. Nature 1988 ; $335: 452-4$.]

[2. Baltimore D. Nature 1988 ; 335 : 395-6.]

La suppression d'un type cellulaire par expression d'un transgène, codant pour une toxine, est un outil puissant en embryologie expérimentale. médecine/sciences a déjà présenté cette technique « d'ablation génétique » (brèves $\mathrm{m} / \mathrm{s} n^{\circ} 9$ vol. $3, p$. 557 et $n^{\circ} 3$ vol. 4, p. 194). Il s'agit de construire un ADN recombinant dans lequel la séquence codant pour la toxine est sous le contrôle d'un promoteur et de ses éléments régulateurs spécifiques d'un type particulier de cellule [1]. Des souris transgéniques exprimant ainsi le gène de la ricine (toxine modifiant l'ARN ribosomal et bloquant ainsi la traduction des ARN messagers) sous le contrôle $\mathrm{du}$ promoteur du gène codant pour la protéine du cristallin $\ll \alpha$ cristalline » naissent sans cristallin constitué et avec de nombreuses autres malformations des structures de l'œil microphtalmie, malformation (structure plissée) de la rétine, présence de matériel de type cristallinien en des sites ectopiques, etc. Cela sug gère que la morphogenèse de l'œil requiert un développement normal du cristallin. On aperçoit immédiatement le parti que l'on pourra tirer de ce type d'approche en embryologie. Comme cela a été discuté dans ces colonnes récemment [2], toute l'embryologie implique des influences réciproques des populations cellulaires qui se succèdent au cours du développement. Les conséquences sur la morphogenèse de la suppression sélective d'une quelconque de ces populations devraient donc renseigner sur son rôle dans la différenciation d'autres structures

[1. Lande PCP, et al. Genes Dev $1988 ; 2$ : 1168-78.]

[2. Chandebois R. médecine/sciences $1988 ; 4$ : 168-76.]

Les anticorps anti-ubiquitine sont plus fréquemment retrouvés dans le lupus érythémateux aigu disséminé (LEAD) que les anticorps anti-ADN natif. Selon une étude du laboratoire de M.H.V. Van Regenmortel à Strasbourg, les pourcentages de positivité de ces deux tests sont respectivement de $79 \%$ et $55 \%$ chez les malades, les faux positifs chez les témoins étant identiques, autour de $3 \%$. La multiplicité des protéines « ubiquitinylées » dans la membrane, le cytoplasme et le noyau $\left(\mathrm{m} / \mathrm{s} n^{\circ} 5\right.$, vol. 2, p. 283 et $n^{\circ} 1$, vol. 4, p. 59) pourrait expliquer la disséminiation de l'atteinte auto-immune dans cette maladie. La détection des anticorps anti-ubiquitine pourrait constituer dans l'avenir un test diagnostique sûr du LEAD.

[Muller S, et al. Proc Natl Acad Sci USA 1988 (sous presse).] 